\title{
Super-Exponential Methods Incorporated with Higher-Order Correlations for Deflationary Blind Equalization of MIMO Linear Systems
}

\author{
Kiyotaka Kohno $^{1}$, Yujiro Inouye ${ }^{1}$, and Mitsuru Kawamoto ${ }^{2}$ \\ 1 Department of Electronic and Control Systems Engineering, Shimane University \\ 1060 Nishikawatsu, Matsue, Shimane 690-8504, Japan \\ kohno@yonago-k.ac.jp; inouye@riko.shimane-u.ac.jp \\ 2 Department of Electronic and Control Systems Engineering, Shimane University \\ 1060 Nishikawatsu, Matsue, Shimane 690-8504, Japan \\ Bio-Mimetic Control Research Center, RIKEN, Moriyama, Nagoya 463-003, Japan \\ kawa@ecs.shimane-u.ac.jp
}

\begin{abstract}
The multichannel blind deconvolution of finite-impulse response (FIR) or infinite-impulse response (IIR) systems is investigated using the multichannel super-exponential deflation methods. In the conventional multichannel super-exponential deflation method [4], the socalled "second-order correlation method" is incorporated in order to estimate the contributions of an extracted source signal to the channel outputs. We propose a new multichannel super-exponential deflation method using higher-order correlations instead of second-order correlations to reduce the computational complexity in terms of multiplications and to accelerate the performance of equalization. By computer simulations, it is shown that the method of using fourth-order correlations is better than the method of using second-order correlations in a noiseless case or a noisy case.
\end{abstract}

\section{Introduction}

Multichannel blind deconvolution has recently received attention in such fields as digital communications, image processing and neural information processing [1],[2].

Recently, Shalvi and Weinstein proposed an attractive approach to singlechannel blind deconvolution called the super-exponential method (SEM) [3]. Extensions of their idea to multichannel deconvolution were presented by Inouye and Tanebe [4], Martone [5], [6], and Yeung and Yau [7]. In particular, Inouye and Tanebe [4] proposed the multichannel super-exponential deflation method (MSEDM) using second-order correlations. Martone [6], and Kawamoto, Kohno and Inouye [8] proposed MSEDM's using higher-order correlations for instantaneous mixtures or constant channel systems. Adaptive versions of multichannel super-exponential algorithms are presented in [9].

In the present paper, we propose a new MSEDM using higher-order correlations for convolutive mixtures or dynamical channel systems, and show the 
effectiveness of the proposed method by computer simulations. Adaptive versions of proposed method will be appeared in a forthcoming paper.

The present paper uses the following notation: Let $Z$ denote the set of all integers. Let $\boldsymbol{C}^{m \times n}$ denote the set of all $m \times n$ matrices with complex components. The superscripts $T, *, H$ and $\dagger$ denote, respectively, the transpose, the complex conjugate, the complex conjugate transpose (Hermitian) and the (Moore-Penrose) pseudoinverse operations of a matrix. Let $i=\overline{1, n}$ stand for $i=1,2, \cdots, n$.

\section{Assumptions and Preliminaries}

We consider an MIMO channel system with $n$ inputs and $m$ outputs as described by

$$
\boldsymbol{y}(t)=\sum_{k=-\infty}^{\infty} \boldsymbol{H}^{(k)} \boldsymbol{s}(t-k)+\boldsymbol{n}(t), \quad t \in Z,
$$

where

$\boldsymbol{s}(t) \quad n$-column vector of input (or source) signals,

$\boldsymbol{y}(t) \quad m$-column vector of channel outputs,

$\boldsymbol{n}(t) \quad m$-column vector of Gaussian noises,

$\boldsymbol{H}^{(k)} m \times n$ matrix of impulse responses.

The transfer function of the channel system is defined by

$$
\boldsymbol{H}(z)=\sum_{k=-\infty}^{\infty} \boldsymbol{H}^{(k)} z^{k}, \quad z \in C .
$$

For the time being, it is assumed for theoretical analysis that the noise term $\boldsymbol{n}(\mathrm{t})$ in (1) is absent.

To recover the source signals, we process the output signals by an $n \times m$ equalizer (or deconvolver) $\boldsymbol{W}(z)$ described by

$$
\boldsymbol{z}(t)=\sum_{k=-\infty}^{\infty} \boldsymbol{W}^{(k)} \boldsymbol{y}(t-k), \quad t \in Z .
$$

The objective of multichannel blind deconvolution is to construct an equalizer that recovers the original source signals only from the measurements of the corresponding outputs.

We put the following assumptions on the systems and the source signals.

A1) The transfer function $\boldsymbol{H}(z)$ is stable and has full column rank on the unit circle $|z|=1$ [ this implies that the unknown system has less inputs than outputs, i.e., $n \leq m$, and there exists a left stable inverse of the unknown system ]. A2) The input sequence $\{\boldsymbol{s}(t)\}$ is a complex, zero-mean, non-Gaussian random vector process with element processes $\left\{s_{i}(t)\right\}, i=\overline{1, n}$ being mutually independent. Moreover, each element process $\left\{s_{i}(t)\right\}$ is an i.i.d. process with a nonzero variance $\sigma_{i}^{2}$ and a nonzero fourth-order cumulant $\gamma_{i}$. The variances $\sigma_{i}^{2}$ 's and the fourth-order cumulants $\gamma_{i}$ 's are unknown.

A3) The equalizer $\boldsymbol{W}(z)$ is an FIR system of sufficient length $L$ so that the truncation effect can be ignored. 
Remark 1: As to A1), if the channel system $\boldsymbol{H}(z)$ is FIR, then a condition of the existence of an FIR equalizer is rank $\boldsymbol{H}(z)=n$ for all nonzero $z \in C$ [10]. Moreover, if $\boldsymbol{H}(z)$ is irreducible, then there exists an equalizer $\boldsymbol{W}(z)$ of length $L \leq n(K-1)$, where $K$ is the length of the channel system [10]. Besides, it is shown that there exists generically (or except for pathological cases) an equalizer $\boldsymbol{W}(z)$ of length $L \leq\left\lceil\frac{n(K-1)}{m-n}\right\rceil$, where $\lceil x\rceil$ stands for the smallest integer that is greater than equal to $x$.

Let us consider an FIR equalizer with the transfer function $\boldsymbol{W}(z)$ given by

$$
\boldsymbol{W}(z)=\sum_{k=L_{1}}^{L_{2}} \boldsymbol{W}^{(k)} z^{k},
$$

where the length $L:=L_{2}-L_{1}+1$ is taken to be sufficiently large. Let $\tilde{\boldsymbol{w}}_{i}$ be the $L m$-column vector consisting of the tap coefficients (corresponding to the $i$ th output) of the equalizer defined by

$$
\begin{aligned}
& \tilde{\boldsymbol{w}}_{i}:=\left[\boldsymbol{w}_{i, 1}^{T}, \boldsymbol{w}_{i, 2}^{T}, \cdots, \boldsymbol{w}_{i, m}^{T}\right]^{T} \in \boldsymbol{C}^{m L}, \\
& \boldsymbol{w}_{i, j}=\left[w_{i, j}{ }^{\left(L_{1}\right)}, w_{i, j}{ }^{\left(L_{1}+1\right)}, \cdots, w_{i, j}{ }^{\left(L_{2}\right)}\right]^{T} \in \boldsymbol{C}^{L},
\end{aligned}
$$

where $w_{i, j}{ }^{(k)}$ is the $(i, j)$ th element of matrix $\boldsymbol{W}^{(k)}$.

Inouye and Tanebe [4] proposed the multichannel super-exponential algorithm for finding the tap coefficient vectors $\tilde{\boldsymbol{w}}_{i}$ 's of the equalizer $\boldsymbol{W}(z)$, of which each iteration consists of the following two steps:

$$
\begin{array}{ll}
\tilde{\boldsymbol{w}}_{i}^{[1]}=\tilde{\boldsymbol{R}}_{L}^{\dagger} \tilde{\boldsymbol{d}}_{i} & \text { for } i=\overline{1, n}, \\
\tilde{\boldsymbol{w}}_{i}^{[2]}=\frac{\tilde{\boldsymbol{w}}_{i}^{[1]}}{\sqrt{\tilde{\boldsymbol{w}}_{i}^{[1] H} \tilde{\boldsymbol{R}}_{L} \tilde{\boldsymbol{w}}_{i}^{[1]}}} \text { for } i=\overline{1, n},
\end{array}
$$

where $(\cdot)^{[1]}$ and $(\cdot)^{[2]}$ stand respectively for the result of the first step and the result of the second step. Let $\tilde{\boldsymbol{y}}(t)$ be the $L m$-column vector consisting of the $L$ consecutive inputs of the equalizer defined by

$$
\begin{aligned}
& \tilde{\boldsymbol{y}}(t):=\left[\overline{\boldsymbol{y}}_{1}(t)^{T}, \overline{\boldsymbol{y}}_{2}(t)^{T}, \cdots, \overline{\boldsymbol{y}}_{m}(t)^{T}\right]^{T} \in \boldsymbol{C}^{m L}, \\
& \overline{\boldsymbol{y}}_{i}(t):=\left[y_{i}\left(t-L_{1}\right), y_{i}\left(t-L_{1}-1\right), \cdots, y_{i}\left(t-L_{2}\right)\right]^{T} \in \boldsymbol{C}^{L},
\end{aligned}
$$

where $y_{i}(t)$ is the $i$ th element of the output vector $\boldsymbol{y}(t)$ of the channel system in (1). Then the correlation matrix $\tilde{\boldsymbol{R}}_{L}$ is represented as

$$
\tilde{\boldsymbol{R}}_{L}=E\left[\tilde{\boldsymbol{y}}^{*}(t) \tilde{\boldsymbol{y}}^{T}(t)\right] \in \boldsymbol{C}^{m L \times m L},
$$

and the fourth-order cumulant vector $\tilde{\boldsymbol{d}}_{i}$ is represented as

$$
\begin{aligned}
\tilde{\boldsymbol{d}}_{i}=E\left[\left|z_{i}(t)\right|^{2} z_{i}(t) \tilde{\boldsymbol{y}}^{*}(t)\right] & -2 E\left[\left|z_{i}(t)\right|^{2}\right] E\left[z_{i}(t) \tilde{\boldsymbol{y}}^{*}(t)\right] \\
& -E\left[z_{i}{ }^{2}(t)\right] E\left[z_{i}{ }^{*}(t) \tilde{\boldsymbol{y}}^{*}(t)\right] \in \boldsymbol{C}^{m L},
\end{aligned}
$$

where $\mathrm{E}[x]$ denotes the expectation of a random variable $x$. We note that the last term can be ignored in case of $\mathrm{E}\left[s_{i}{ }^{2}(t)\right]=0$ for all $i=\overline{1, n}$, in which case $\mathrm{E}\left[z_{i}{ }^{2}(t)\right]=0$ for all $i=\overline{1, n}$. 


\section{A Super-Exponential Deflation Method Incorporated with Higher-Order Correlations}

The MSEDM proposed by Inouye and Tanebe [4] uses second-order correlations to estimate the contributions of an extracted source signal to the channel outputs. We utilize higher-order correlations instead of second-order correlations in order to estimate the contributions of an extracted source signal to the channel outputs. For notational simplicity, we confine ourselves to fourth-order correlations although our results are expandable to higher-order correlations.

For the details of the method of second-order correlations, see the equations from (55) through (58) in [4]. According to the discussions from (55) through (58) in [4], we calculate the higher-order cross-correlations of the equalizer outputs $z_{i}(t)$ 's with the channel outputs $y_{k}(t)$ 's, and define a possibly scaled and timeshifted estimate of the channel element $h_{k, j_{i}}(\tau)$ as

$$
\hat{h}_{k, j_{i}}:=\operatorname{cum}\left(z_{i}^{*}(t-\tau), z_{i}^{*}(t-\tau), z_{i}(t-\tau), y_{k}(t)\right) .
$$

Then let us consider the contribution of $z_{i}(t)$ to the channel output $y_{k}(t)$ which is defined by

$$
\hat{y}_{k, j_{i}}(\tau):=\frac{\sigma_{j_{i}}^{2}}{\gamma_{j_{i}}} \sum_{\tau} \hat{h}_{k, j_{i}}(\tau) z_{i}(t-\tau),
$$

where $\frac{\sigma_{j_{i}}^{2}}{\gamma_{j_{i}}}$ is introduced to adjust the difference between the scale of the contribution of $z_{i}(t)$ and the scale of the contribution of source $s_{j_{i}}(t)$ to the channel output $y_{k}(t)$. Subtract the above contribution from the channel output $\boldsymbol{y}(t)$ as

$$
y_{k}^{(i)}(t):=y_{k}(t)-\hat{y}_{k, j_{i}}(t), k=\overline{1, m} .
$$

Let us analyze the above method. After the first cycle of the iteration, the first equalizer output $z_{1}(t)$ is a possibly scaled and time-shifted version of one of the channel input, that is,

$$
z_{1}(t)=d s_{j_{1}}\left(t-k_{1}\right), j_{1} \in\{1,2, \cdots, n\},
$$

where $|d|=1 / \sigma_{j_{1}}$ and $k_{1}$ represents a delay-time, which may belong to the interval $\left[K+L_{1}, L_{2}\right]$ (see (28) for the derivation of the above relation), i.e.,

$$
k_{1} \in\left[K+L_{1}, L_{2}\right],
$$

in the case when the channel $\boldsymbol{H}$ is an FIR system with $\{\boldsymbol{H}(k)\}_{0}^{K-1}$, and the equalizer $\boldsymbol{W}$ is an FIR system with $\{\boldsymbol{W}(k)\}_{k=L_{1}}^{L_{2}}$.

Since

$$
\begin{array}{r}
\operatorname{cum}\left(z_{i}^{*}(t-\tau), z_{i}^{*}(t-\tau), z_{i}(t-\tau), y_{k}(t)\right) \\
=\operatorname{cum}\left(z_{i}^{*}(t), z_{i}^{*}(t), z_{i}(t), y_{k}(t+\tau)\right) .
\end{array}
$$

it follows from (13)(with $i=1$ ) and (16)

$$
\begin{aligned}
\hat{h}_{k . j_{1}}(\tau) & =\operatorname{cum}\left(z_{1}^{*}(t), z_{1}^{*}(t), z_{1}(t), y_{k}(t+\tau)\right) \\
& =\sum_{j=1}^{n} \sum_{l} h_{k, j}(l) \operatorname{cum}\left(z_{1}^{*}(t), z_{1}^{*}(t), z_{1}(t), s_{j}(t+i-l)\right)
\end{aligned}
$$




$$
\begin{aligned}
& =d^{* 2} d \sum_{j=1}^{n} \sum_{l} h_{k, j}(l) \operatorname{cum}\left(s_{j_{1}}^{*}\left(t-k_{1}\right), s_{j_{1}}^{*}\left(t-k_{1}\right), s_{j_{1}}\left(t-k_{1}\right), s_{j}(t+\tau-l)\right) \\
& =d^{* 2} d h_{k, j_{1}}\left(k_{1}+\tau\right) \gamma_{j_{1}},
\end{aligned}
$$

which means

$$
\hat{h}_{k . j_{1}}(\tau)=|d|^{2} d^{*} \gamma_{j_{1}} h_{k, j_{1}}\left(k_{1}+\tau\right) .
$$

Substituting (16) and (19) into (14)(with $i=1$ ) gives

$$
\begin{aligned}
\hat{y}_{k, j_{1}}(t) & =\frac{\sigma_{j_{1}}^{2}}{\gamma_{j_{1}}} \sum_{\tau}|d|^{4} d^{*} \gamma_{j_{1}} h_{k, j_{1}}\left(k_{1}+\tau\right) s_{j_{1}}\left(t-\tau-k_{1}\right) \\
& =\sum_{\tau} h_{k, j_{1}}\left(k_{1}+\tau\right) s_{j_{1}}\left(t-\tau-k_{1}\right) \\
& =\sum_{\tau} h_{k, j_{1}}(\tau) s_{j_{1}}(t-\tau) .
\end{aligned}
$$

Thus, we obtain from (15)

$$
\begin{aligned}
y_{k}^{(1)}(t) & =y_{k}(t)-\hat{y}_{k, j_{1}}(t) \\
& =\sum_{j=1}^{n} \sum_{\tau} h_{k, j}(\tau) s_{j}(t-\tau)-\sum_{\tau} h_{k, j_{1}}(\tau) s_{j_{1}}(t-\tau) \\
& =\sum_{j=1, j \neq j_{1}} h_{k, j}(\tau) s_{j}(t-\tau),
\end{aligned}
$$

which shows that $y_{k}^{(1)}(t)$ does not contains the contribution of the source $s_{j_{1}}(t)$.

As one of the advantages of the above method, we can reduce the computational loads for calculating (13) and (14) as follows: Using the definitions of $\hat{h}_{k, j_{i}}$ and $\left[d_{i, j}\right]_{\tau}$ (see (13) and (44) in $[4]$ ), we have

$$
\hat{h}_{k, j_{i}}(\tau)=\left[d_{i, k}\right]_{-\tau}^{*},
$$

Therefore, (14) becomes

$$
\begin{aligned}
\hat{y}_{k, j_{i}}(t) & =\frac{\sigma_{j_{i}}^{2}}{\gamma_{j_{i}}} \sum_{\tau} \hat{h}_{k, j_{i}} z_{i}(t-\tau) \\
& =\frac{\sigma_{j_{i}}^{2}}{\gamma_{j_{i}}} \sum_{\tau}\left[d_{i, k}\right]_{\tau}^{*} z_{i}(t+\tau) \\
& =\frac{\sigma_{j_{i}}^{2}}{\gamma_{j_{i}}} \sum_{\tau=L_{1}}^{L_{2}}\left[d_{i, k}\right]_{\tau}^{*} z_{i}(t+\tau) .
\end{aligned}
$$

Thus, (15) becomes

$$
y_{k}^{(i)}(t)=y_{k}(t)-\frac{\sigma_{j_{i}}^{2}}{\gamma_{j_{i}}} \sum_{\tau=L_{1}}^{L_{2}}\left[d_{i, k}\right]_{\tau}^{*} z_{i}(t+\tau), k=\overline{1, m},
$$

where the coefficients $\left[d_{i, k}\right]_{\tau}$ 's are available at the first step (7) of the two-step iteration.

Some remarks are given below on conditions for the indices $L_{1}$ and $L_{2}$ of the equalizer. In the following discussion, we assume that delay-time $k_{1}$ in (16) is 
known or estimated ahead. If follows from (19) and (22) that

$$
h_{k, j_{1}}\left(\tau+k_{1}\right)=\frac{1}{\alpha} \hat{h}_{k, j_{1}}(\tau)=\frac{1}{\alpha}\left[d_{i, j}\right]_{-\tau}^{*},
$$

where $\alpha=\left|d^{2}\right| d^{*} \gamma_{j_{1}}$. When the channel $\boldsymbol{H}$ is an FIR system with $\{\boldsymbol{H}(k)\}_{0}^{K-1}$ and the equalizer $\boldsymbol{W}$ is an FIR system with $\{\boldsymbol{W}(k)\}_{k=L_{1}}^{L_{2}}$, the support of the function $h_{k, j_{1}}(\tau)$ is the interval $[0, K]$ and the support of the function $\left[d_{i, j}\right]_{\tau}$ is the interval $\left[L_{1}, L_{2}\right]$. Here, given a function $h(\tau)$ defined on $Z$, the subset $\{\tau \in Z: h(\tau) \neq 0\}$ is called the support of the function $h$. Therefore, in order for the sequence $\left[d_{i, j}\right]_{-\tau}^{*}$ to determine the values of the sequence $h_{k, j_{1}}\left(\tau+k_{1}\right)$ based on (25), the support of $h_{k, j_{1}}\left(\tau+k_{1}\right)$ should be included in the support of $\left[d_{i, j}\right]_{-\tau}^{*}$, that is,

$$
\left[-k_{1},-k_{1}+K\right] \subset\left[-L_{2},-L_{1}\right]
$$

which implies

$$
L_{1} \leq k_{1}-K, L_{2} \geq k_{1}
$$

or

$$
k_{1} \in\left[L_{1}+K, L_{2}\right] .
$$

Thus the first tap index $L_{1}$ and the last tap index $L_{2}$ of the equalizer are chosen to satisfy the conditions

$$
\begin{aligned}
& L_{1} \leq k_{1}-K, \\
& L_{2} \geq k_{1} .
\end{aligned}
$$

\section{Simulations}

To demonstrate the effectiveness of the proposed method, many computer simulations were conducted. We considered an MIMO channel system with two inputs and three outputs, and assumed that the length of the channel is three $(K=3)$, that is, $\boldsymbol{H}^{(k)}$ in (1) was set to be

$$
\boldsymbol{H}(z)=\left[\begin{array}{cc}
1.00+0.60 z+0.30 z^{2} & 0.60+0.50 z-0.20 z^{2} \\
0.50-0.10 z+0.20 z^{2} & 0.30+0.40 z+0.50 z^{2} \\
0.70+0.10 z+0.40 z^{2} & 0.10+0.20 z+0.10 z^{2}
\end{array}\right] .
$$

The length of the equalizer was chosen to be twelve $(L=12)$. We set the values of the tap coefficients to be zero except for $w_{12}(6)=w_{22}(6)=1$. Two source signals were the 4-PSK and the 8-PSK signals, respectively. Three independent Gaussian noises (with identical variance $\sigma_{w}^{2}$ ) were added to the three outputs $y_{i}(t)$ 's at various SNR levels. The SNR is defined as SNR: $=10 \log _{10}\left(\sigma_{i}^{2} / \sigma_{w}^{2}\right)$, where $\sigma_{i}^{2}$ 's are the variances of $s_{i}(t)$ 's and are equal to one. As a measure of performance, we use the multichannel intersymbol interference $\left(\mathrm{M}_{\mathrm{ISI}}\right)$ defined in the logarithmic $(\mathrm{dB})$ scale by

$$
\mathrm{M}_{\mathrm{ISI}}:=10 \log _{10}\left[\sum_{i=1}^{n} \frac{\left.\left|\Sigma_{j=1}^{n} \Sigma_{t=-\infty}^{\infty}\right| g_{i, j}(t)\right|^{2}-\left|g_{i, \cdot}\right|_{\max }^{2} \mid}{\left|g_{i, \cdot}\right|_{\max }^{2}}\right.
$$




$$
\left.+\sum_{j=1}^{n} \frac{\left.\left|\Sigma_{i=1}^{n} \Sigma_{t=-\infty}^{\infty}\right| g_{i, j}(t)\right|^{2}-\left|g_{\cdot, j}\right|_{\max }^{2} \mid}{\left|g_{\cdot, j}\right|_{\max }^{2}}\right],
$$

where $\left|g_{i,}\right|_{\max }^{2}$ and $\left|g_{, j}\right|_{\text {max }}^{2}$ are respectively defined by

$$
\left|g_{i, \cdot}\right|_{\max }^{2}:=\max _{j=1,2}\left|g_{i, j}\right|^{2},\left|g_{\cdot, j}\right|_{\max }^{2}:=\max _{i=1,2}\left|g_{i, j}\right|^{2} .
$$

Fig. 1 shows the averages of performance results over 10 independent Monte Carlo runs for the proposed method and the conventional method [4] in the noiseless case when the time-duration is from zero to 30 (1000 iterations were carried out in each time-duration). In each Monte Carlo run, $\tilde{\boldsymbol{R}}_{L}$ was estimated using 1,000 data samples. In each iteration of two steps (7) and (8), $\tilde{\boldsymbol{d}}_{i}$ was estimated using 1,000 data samples. It can be seen from Fig. 1 that our proposed method is better than the conventional method about $7 \mathrm{~dB}$ when the second source signal is deconvolved.

Fig. 2 shows the averages of the performance results over 5 independent Monte Carlo runs when the SNR level was taken to be $0[\mathrm{~dB}], 5[\mathrm{~dB}], 10[\mathrm{~dB}]$, $15[\mathrm{~dB}], 20[\mathrm{~dB}]$ and $\infty[\mathrm{dB}]$, respectively. It can be seen from Fig. 2 that our proposed method is superior to the conventional method in the noiseless case, and our proposed method works as well as the conventional method even when the power of additive noise increases.

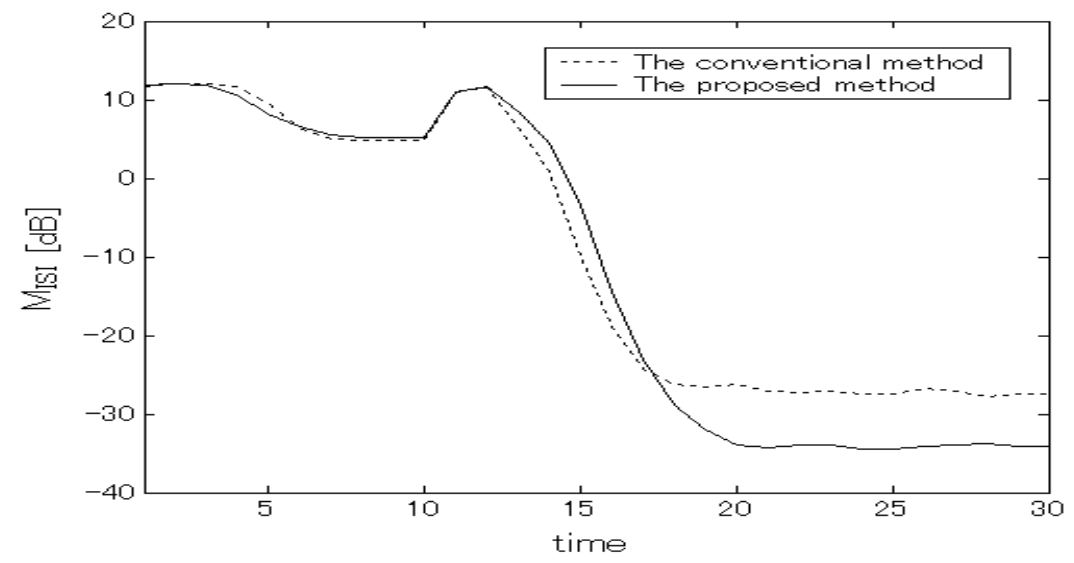

Fig. 1. Comparison between the proposed method and the conventional method (Noiseless case)

\section{Conclusions}

We have proposed a new multichannel super-exponential deflation method using higher-order correlations instead of second-order correlations for estimating the contributions of an extracted source signal to the channel outputs in order to reduce the computational complexity and to accelerate the performance of equalization. By computer simulations, it is shown that the method of using fourth-order correlations is superior to the method of using second-order correlations in a noiseless case or a noisy case. 


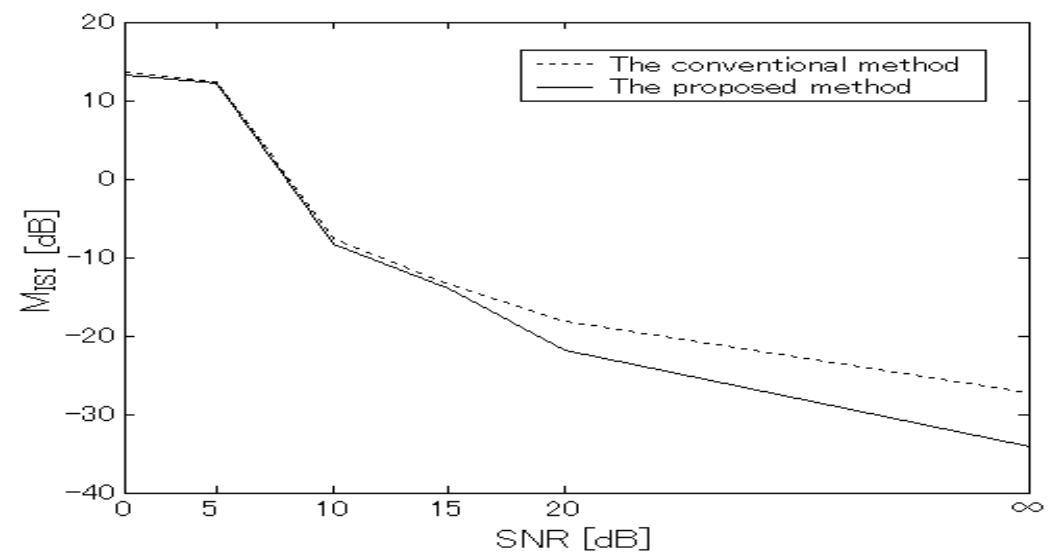

Fig. 2. Comparison between the proposed method and the conventional method (Noisy case)

\section{References}

1. Special issue on blind system identification and estimation, Proc. IEEE, vol. 86, no. 10, pp. 1907-2089, Oct. 1998.

2. Special section on blind signal processing: Independent component analysis and signal separation, IEICE Trans. on Fundamentals of Electronics, vol. EA86A, no. 3, pp. 522-642, Mar. 2003.

3. O. Shalvi and E. Weinstein, "Super-exponential methods for blind deconvolution," IEEE Trans. Information Theory, vol. 39, no. 2, pp. 504-519, Mar. 1993.

4. Y. Inouye and K. Tanebe, "Super-exponential algorithms for multichannel blind deconvolution," IEEE Trans. Signal Processing, vol. 48, no. 3, pp. 881-888, Mar. 2000.

5. M. Martone, "An adaptive algorithm for antenna array low-rank processing in cellular TDMA base stations," IEEE Trans. Communications, vol. 46, no. 5, pp. 627-643, May 1998.

6. M, Martone, "Fast adaptive super-exponential multistage beamforming for cellular base-station transceivers with antenna arrays," IEEE Trans. Vehicular Tech., vol. 48, no. 4, Jul. 1999.

7. K. L. Yeung and S. F. Yau, "A cumulant-based super-exponential algorithm for blind deconvolution of multi-input multi-output systems," Signal Process., vol. 67, pp. 141-162, 1998.

8. M. Kawamoto, K. Kohno and Y. Inouye, "Robust Super-Exponential Methods for Deflationary Blind Equalization of Instantaneous Mixtures," submitted to IEEE Trans. Signal Processing.

9. K. Kohno, Y. Inouye, M. Kawamoto and Tetsuya Okamoto, "Adaptive SuperExponential Algorithms for Blind Deconvolution of MIMO Systems," accepted for presentation in ISCAS 2004.

10. Y. Inouye and R-W. Liu, "A system-theoretic foundation for blind equalization of an FIR MIMO channel system," IEEE Trans. Circuits and Systems - 1, Fundam. Theory Appl., vol. 49, no. 4, pp. 425-436, Apr. 2002. 